\title{
Generation and Transmission Expansion Planning With Respect to Global Warming Potential
}

This paper was downloaded from TechRxiv (https://www.techrxiv.org).

\section{LICENSE}

CC BY 4.0

SUBMISSION DATE / POSTED DATE

20-04-2021 / 22-04-2021

CITATION

Schwaeppe, Henrik; Moser, Albert; Paronuzzi, Paolo; Monaci, Michele (2021): Generation and Transmission Expansion Planning With Respect to Global Warming Potential. TechRxiv. Preprint. https://doi.org/10.36227/techrxiv.14453694.v1

DOI

10.36227/techrxiv.14453694.v1 


\section{Generation and Transmission Expansion Planning With Respect to Global Warming Potential}

\author{
Henrik Schwaeppe, Albert Moser \\ Institute of High Voltage Equipment and Grids, Digitalization \\ and Energy Economics (IAEW), RWTH Aachen University \\ Aachen, Germany \\ henrik.schwaeppe@rwth-aachen.de
}

\author{
Paolo Paronuzzi, Michele Monaci \\ DEI "Guglielmo Marconi" \\ University of Bologna \\ Bologna, Italy \\ \{michele.monaci, paolo.paronuzzi\}@unibo.it
}

\begin{abstract}
As a result of global efforts to reduce greenhouse gas emissions, future energy system designs must be investigated. However, previous studies in integrated generation and transmission expansion planning neglect indirect emissions and therefore the full global warming potential of system designs. We introduce a linear, single-stage integrated expansion planning model with respect to global warming potential of all components. Neglecting indirect emissions underestimates the global warming potential by more than $20 \%$ and the total cost by almost $10 \%$ in an exemplary showcase. The inclusion of indirect emissions tightens the emission budget and therefore leads to different system designs.
\end{abstract}

Index Terms - transmission expansion planning, generation expansion planning, emissions, linear programming, global warming potential

\section{INTRODUCTION}

In the course of achieving global carbon net neutrality, renewable energies will foreseeably dominate energy supply. While renewable's supply conditions are more favourable in particular environments (e.g. closer to the equator or in windy coastal areas), high energy demand typically exists away from those potent areas. For this reason the question arises whether it is more ecological and economical to transport energy from distant production locations or to deal with below-optimal energy supply conditions. To find answers, generation expansion planning (GEP) must be enhanced by locational planning and an expandable transmission grid in order to investigate future system designs. Models of this type are subordinated to generation and transmission expansion planning (G\&TEP). With ongoing efforts to mitigate global warming and therefore reduce greenhouse gas (GHG) emissions, the energy sector has seen a strong emergence of published research within G\&TEP.

However, with few exceptions, recent publications only track direct emissions in power supply to ensure consistency with climate targets [1]-[6], not paying attention to the indirect

This research is part of the project PlaMES (Integrated Planning of MultiEnergy Systems). PlaMES has received funding from the European Union's Horizon 2020 research and innovation programme under grant agreement No. 863922. The content of this publication reflects only the authors' view. The Innovation and Networks Executive Agency (INEA) is not responsible for any use that may be made of the information it contains.
GHG emissions along the life-cycle of components 1 Indirect emissions make up the majority of the total emissions of renewable energy technologies (RETs) [7]. Neither will RETs reach close-to-zero life-cycle GHG emissions in the future [8], nor will additionally required energy storages be emission-free through their life-cylce. While carbon net neutrality does not imply zero emissions, the average annual absorption of carbon sinks defines a strict emission limit. Hence, the inclusion of indirect emissions - obtained from life-cycle assessment (LCA) studies - could have a restrictive and probably costly effect on finding efficient and sustainable energy system designs. Therefore the goal of this paper is to investigate first implications of life-cycle indirect emissions in G\&TEP models.

After outlining emissions and LCA in the context of energy system modelling in Section II] we introduce a linear single-stage G\&TEP model that incorporates life-cycle GHG emissions in Section III A sensitivity study on an exemplary test case (presented in Section IV) showcases the impact of life-cycle GHG emissions on finding efficient future energy system designs in Section $\mathrm{V}$

\section{CONTEXT}

Different approaches to emissions exist within related literature. While the inclusion of direct emissions has become standard in GEP problems, TEP problems typically neglect emissions. Among direct emissions in G\&TEP models, studies can be distinguished between GHG emissions [1], [5] and emissions related to health or nature conservation, like pollution, sulphur oxides (SOx) or nitrogen oxides (NOx) [2]. Other studies fully neglect emissions or do not reveal details about their inclusion [3]. Very few studies include indirect emissions into their direct emission parameter to reflect life-cycle impacts [9]. Recently, only one study has differentiated between direct and indirect emissions [6]. So far no study includes the global warming potential (GWP) of static components such as transformers, lines or cables. Other parameters to reach environment-, health- or sustainability-

\footnotetext{
${ }^{1}$ By direct emissions we mean operational emissions, e.g. from burning fuel. Indirect emissions include emissions from production, construction, maintenance, repair, recycling etc.
} 
related targets could be monitored as well, but are outside the scope of this paper.

In LCA studies, global warming potential is typically given in kilograms or tons of $\mathrm{CO}_{2}$-equivalents per unit. Given units do not always allow for adoption into an energy system model (e.g. per kilogram material) or don't deduct unneeded information (GWP of transmission grid at current energy mix). Researching literature values for life-cycle emissions, we also realized that reliable data about life-cycle GHG emissions are either sparse or very specific and show incoherent variety among different studies and data sets. In literature, emission intensity varies depending on the year of study, location of production and inherently the carbon-intensity of energy supply. Conducted LCA studies mostly investigate the status quo of production and do not give any insight about future potentials, like the decreasing carbon intensity of energy supply, replacement of GHGs in transformers, scale of production, new resource-extraction methods etc. Table I provides an excerpt of LCA studies we have consulted for this paper. We acknowledge that including life-cycle data adds another dimension of uncertainty to the model parameters that cannot be countered at the current state of information. On the other hand, neglecting indirect emissions cannot be considered good practice either.

TABLE I

EXEMPLARY GLOBAL WARMING POTENTIAL IN LITERATURE: MINIMAL, AVERAGE AND MAXIMAL VALUES OF TODAY AND FUTURE OUTLOOK

\begin{tabular}{|c|c|c|c|c|}
\hline \multirow[t]{2}{*}{ Technology } & minimal & average & maximal & $2050 \sqrt{8}$ \\
\hline & \multicolumn{4}{|c|}{$\left[\mathrm{kg} \mathrm{CO}_{2}\right.$-eq/MWh] } \\
\hline Photovoltaic [10], |11] & 8 & 32 & 80 & $4(3-21)$ \\
\hline Onshore wind $\mid 11$ & 5 & 8 & 25 & $4(3-6)$ \\
\hline Offshore wind $|\overline{12}|,|\overline{13}|$ & 7 & 8 & 16 & $4(3-0)$ \\
\hline \multirow[t]{2}{*}{ Stationary battery $[14]^{a, b}$} & 10 & I & 180 & I \\
\hline & \multicolumn{4}{|c|}{ [t $\mathrm{CO}_{2}$-eq/MVA] } \\
\hline Photovoltaic [15] & 824 & 1280 & 2870 & I \\
\hline \multirow[t]{2}{*}{ Transformer [16], |17] } & 9 & l & 119 & I \\
\hline & \multicolumn{4}{|c|}{$\left[\mathrm{t} \mathrm{CO}_{2}-\mathrm{eq} / \mathrm{km}\right]$} \\
\hline Overhead line [18] & l & 135 & 1 & l \\
\hline Underground cable $[18]$ & l & 632 & l & l \\
\hline
\end{tabular}

\section{MODEL}

In the following, a mathematical model for the singlestage generation and transmission expansion planning model is

TABLE II

SETS USED WITHIN THE MODEL.

\begin{tabular}{ll} 
Set & Description \\
\hline$N=\{1, \ldots, n\}$ & Set of nodes \\
$E=\{1, \ldots, m\}$ & Set of edges (transmission lines) \\
$T=\{1, \ldots, \theta\}$ & Set of time slots \\
$I=\{W, O, P, B, F\}$ & Set of technologies: Wind, Offshore, \\
& Photovoltaic, Batteries, Fuel power plants \\
$M(j) \subset I$ & Subset of technologies available at node $j$ \\
$E_{\text {cand }} \subset E$ & Set of transmission candidate lines
\end{tabular}

described. The model is purely linear and intended to provide a general framework for multi-energy planning. The singleobjective function described by (1) aims to reduce total system cost of operation, installation, transmission and fuel as well as curtailment and - required to find a valid solution - energy provision through slack in compliance with the GHG emission constraint (7). As the goal is to find GHG compliant energy system designs - not to determine taxes at which compliant systems would be achieved - an emission cap is preferred instead of an emission tax (which would also result in a multiobjective function). Used sets, variables and parameters can be taken from Table II to IV.

$$
\begin{aligned}
& \min C_{O P}+C_{I N S T}+C_{T R M}+C_{F U E L}+C_{S L K} \\
& \text { s.t. } C_{O P}=\sum_{t \in T} \sum_{j \in N: B \in M(j)} C_{B j}^{p}\left(x_{B j t}^{d}-x_{B j t}^{c}\right) \\
& C_{I N S T}=\sum_{j \in N} \sum_{i \in M(j)} C_{i j}^{C} y_{i j}+\sum_{g \in E_{\text {cand }}} C_{g}^{V} l_{g} \\
& C_{T R M}=\sum_{t \in T} \sum_{e \in E} c_{e t}^{T}+\sum_{t \in T} \sum_{g \in E_{\text {cand }}} c_{g t}^{V P I} \\
& C_{F U E L}=\sum_{j \in N: F \in M(j)} C_{F j}^{p} \frac{x_{F j t}}{\eta_{F j}} \\
& C_{S L K}=\sum_{t \in T} \sum_{j \in N}\left(C_{j}^{+} s_{j t}^{+}+C_{j}^{-} s_{j t}^{-}\right) \\
& \sum_{j \in N} \sum_{i \in M(j)} e_{i j}+\sum_{g \in E_{\text {cand }}} e_{g} \leq G H G_{t o t} .
\end{aligned}
$$

As a general concept, the electric output of any technology $i$ at an electric node $j$ during time slot $t$ can be described by a variable $x_{i j t}$ and is limited by the installed capacity $y_{i j}$. The expansion can be capped at a defined regional/nodal limit by $Y_{i j}^{\max }$ (e.g. required for expansion potentials):

$$
0 \leq x_{i j t} \leq y_{i j} \leq Y_{i j}^{\max }
$$

Further, electric demand is described by $D_{j t}<0$. Slack variables are defined to tackle overdemand and oversupply in (9) and (10), respectively.

$$
\begin{array}{r}
0 \leq s_{j t}^{+} \leq-D_{j t} \\
s_{j t}^{-} \leq 0
\end{array}
$$

With former values, nodal power injection in the grid of node $j$ during time slot $t$ is described as:

$$
z_{j t}=\sum_{i \in M(j)} x_{i j t}+D_{j t}+s_{j t}^{+}+s_{j t}^{-} .
$$

\section{A. Transmission network expansion planning}

To reflect constraints in the transmission network we use a power transfer distribution factor (PTDF) approach. Transmission line expansion is added through virtual power injection (VPI) as suggested in [19], but with relaxed binary variables to allow for partial expansion. The PTDF coefficients, denoted 
TABLE III

VARIABLES USED WITHIN THE MODEL

\begin{tabular}{ll} 
Variable & Description \\
\hline$x_{i j t}$ & $\begin{array}{l}\text { Amount of electric power generated / withdrawn by technol- } \\
\text { ogy } i \in I \text { in node } j \in N \text { during time slot } t \in T\end{array}$ \\
$z_{j t}$ & Power injection at node $j \in N$ during time slot $t \in T$ \\
$y_{i j}$ & Installed capacity at node $j \in N$ for technology $i \in M(j)$ \\
$l_{g}$ & Installed fraction of candidate line $g \in E_{\text {cand }}$ \\
$v_{g t}$ & Virtual injection of candidate edge $g \in E_{\text {cand }}$ at $t \in T$ \\
$s_{j t}^{-}$ & Curtailed power at node $j \in N$ during time slot $t \in T$ (due \\
& to oversupply / congestion) \\
$s_{j t}^{+}$ & Power deficit slack at node $j \in N$ during time slot $t \in T$ \\
$c_{e t}^{T}$ & Transmission cost for edge $e \in E$ during time slot $t \in T$ \\
$c_{g t}^{V} V$ & Virtual power injection cost at edge $g \in E_{\text {cand }}$ at $t \in T$ \\
$e_{i j}$ & Emissions at node $j \in N$ and technology $i \in M(j)$ \\
$e_{g}$ & Emissions related to installation of candidate line $g \in E_{c a n d}$ \\
$f_{e t}$ & Power flow along edge $e \in E$ during time slot $t \in T$ \\
$x_{B j t}^{c}$ & Amount of energy that is used to charge a battery at node \\
& $j \in N$ s.t. $B \in M(j)$ during time slot $t \in T$ \\
$x_{B j t}^{d}$ & Amount of energy that is used to discharge a battery at node \\
& $j \in N$ s.t. $B \in M(j)$ during time slot $t \in T$ \\
$w_{B j}^{m a x}$ & Maximal enegy storage at node $j \in N$ s.t. $B \in M(j)$ \\
$h_{B j t}$ & Energy stored at node $j \in N$ s.t. $B \in M(j)$ at $t \in T$
\end{tabular}

TABLE IV

PARAMETERS USED WITHIN THE MODEL.

\begin{tabular}{|c|c|}
\hline Parameter & escription \\
\hline$Y_{i j}^{\max }$ & Maximal capacity at node $j \in N$ for technology $i \in M(j)$ \\
\hline$D_{j t}$ & Electric demand at node $j \in N$ in time slot $t \in T$ \\
\hline$\eta_{B j}^{c}$ & Battery charge efficiency at node $j \in N$ s.t. $B \in M(j)$ \\
\hline$\eta_{B j}^{d}$ & Battery discharge efficiency at node $j \in N$ s.t. $B \in M(j)$ \\
\hline$\eta_{F j}$ & Energy conversion efficiency at node $j \in N$ s.t. $F \in M(j)$ \\
\hline$C_{i j}^{C}$ & Capacity installation cost of $i \in I$ at node $j \in N$ \\
\hline$C_{g}^{V}$ & Expansion cost to build candidate line $e \in E_{\text {cand }}$ \\
\hline$C_{B j}^{p}$ & Cost to (dis)charge battery at node $j \in N$ s.t. $B \in M(j)$ \\
\hline$C_{F j}^{\vec{p}}$ & Power plant operation cost at node $j \in N$ s.t. $F \in M(j)$ \\
\hline$C_{e}^{T}$ & Unit transmission cost per edge $e \in E$ \\
\hline$C_{j}^{+}$ & Cost of positive power slack at node $j \in N$ \\
\hline$C_{i}^{-}$ & Costs of negative power slack at node $j \in N$ \\
\hline$C_{g}^{V P I}$ & Cost of virtual power injection at candidate line $g \in E_{c}$ \\
\hline$E_{i j}^{g}$ & Operation emission coefficient of $i \in I$ at node $j \in N$ \\
\hline$E_{i j}^{y}$ & Indirect emission coefficient of $i \in I$ at node $j \in N$ \\
\hline$E_{g}^{l}$ & Indirect emission coefficient of new line $g \in E_{\text {cand }}$ \\
\hline$F_{e}$ & \\
\hline$\Psi_{e j}$ & $\begin{array}{l}\text { PTDF coefficient representing the load on edge } e \in E \text { per } \\
\text { power unit from node } j \in N\end{array}$ \\
\hline$R E N_{i j t}$ & $\begin{array}{l}\text { Normalized feed-in at node } j \in N \text { s.t. } i \in M(j) \cap \\
\{W, O, P\} \text { at time } t \in T\end{array}$ \\
\hline$C A P_{B} j$ & $\begin{array}{l}\text { Capacity of storage of a battery at node } j \in N \text { s.t. } B \in \\
M(j) \text { in relation to its power output }\end{array}$ \\
\hline$C A$ & Initial capacity of storage in relation to its power output \\
\hline & eneroy at node $i$ \\
\hline & \\
\hline
\end{tabular}

as $\Psi$ in the following, are calculated with all candidates built. Every candidate line can be supplemented through VPI.

Candidate lines have two virtual generators. A candidate line $g=(u, w) \in E_{\text {cand }}$ connects two nodes $u, w \in N$. For each $t \in T$, the virtual generators attached to each endpoint of the line are described as $v_{g t}=v_{g t}^{+}=-v_{g t}^{-}$. Virtual generators manipulate the flow on a line. If a line is fully built, virtual generators are idle. If a line is not built, virtual generators inject power in such a way, that the flow equation of the candidate line equals zero, which is equivalent to the line not being built. The virtual generators appear as yet another generator in the flow equation (12) for any line $e \in E$ and time $t \in T$ as follows:

$$
f_{e t}=\sum_{j \in N} \Psi_{e j} z_{j t}+\sum_{g=(u, w) \in E_{\text {cand }}}\left(\Psi_{g u}-\Psi_{g w}\right) v_{g t}
$$

Power flow is constrained by the thermal line capacity $F_{e}$ in (13), and the absolute values are subject to transport cost in (14). This resembles potential losses through otherwise neglected ohmic resistance and rewards localized solutions.

$$
\begin{aligned}
-F_{e} \leq f_{e t} & \leq F_{e} \\
-c_{e t}^{T} \leq C_{e}^{T} f_{e t} & \leq c_{e t}^{T}
\end{aligned}
$$

Whether a candidate line $g=(u, w)$ is built between $u$ and $w$ is dependent on decision variable $l_{g}$. If the line is fully built $\left(l_{g}=1\right)$, the associated virtual generators are inoperable; otherwise they are restricted as described in (15) and (16).

$$
\begin{array}{r}
0 \leq l_{g} \leq 1 \\
F_{g}\left(l_{g}-1\right) \leq v_{g t} \leq F_{g}\left(1-l_{g}\right)
\end{array}
$$

Power flow on a candidate line $g$ is now restricted as in 17 and (18).

$$
\begin{aligned}
& v_{g t}-\left[\sum_{j \in N} \Psi_{g j} z_{j t}+\sum_{g^{\prime} \in E_{\text {cand }}}\left(\Psi_{g^{\prime} u}-\Psi_{g^{\prime} w}\right) v_{g^{\prime} t}\right] \leq l_{g} F_{g} \\
& v_{g t}-\left[\sum_{j \in N} \Psi_{g j} z_{j t}+\sum_{g^{\prime} \in E_{\text {cand }}}\left(\Psi_{g^{\prime} u}-\Psi_{g^{\prime} w}\right) v_{g^{\prime} t}\right] \geq-l_{g} F_{g}
\end{aligned}
$$

In principle, the expansion variables associated with virtual generators are binary. Relaxing this requirement gives more degrees of freedom, especially when a line is built only partially. To favour correct minimal usage of virtual generators, marginal cost is added to VPI usage:

$$
-c_{g t}^{V P I} \leq C_{g}^{V P I} v_{g t} \leq c_{g t}^{V P I} .
$$

From a mathematical point of view, additional transformer capacity can be modelled just as candidate lines. The expansion of grid infrastructure does not only incur cost, but also emissions as:

$$
e_{g}=E_{g}^{l} l_{g} .
$$

\section{B. Generation expansion}

In the following, we assume that system components will be newly built. Existing power plants could be modelled by constraining the investment variable $y_{i j}$ through a lower bound, but will be disregarded in this paper. 
1) Wind turbines and photovoltaic systems: The input from wind turbines and photovoltaic systems is modelled via normalized feed-in profiles $R E N_{i j t}$ imposing constraints for each $t \in T, j \in N$ and $i \in M(j) \cap\{W, O, P\}$ as

$$
x_{i j t} \leq R E N_{i j t} y_{i j} .
$$

Emissions occur through direct operation $\left(E_{i j}^{x}\right)$ or installation $\left(E_{i j}^{y}\right)$ :

$$
e_{i j}=E_{i j}^{y} y_{i j}+\sum_{t \in T} E_{i j}^{x} x_{i j t} .
$$

2) Thermal power plants, fuel cells: The modelling of electrical feed-in from power plants is straightforward. Their output is already described by (8) with a respective cost $C_{F j}^{p}$. Ramps or similar features are neglected for the time being. Both, operation and installation, may incur emissions as described by (22).

3) Electrical Storage: Storage, which is denoted with technology index B in the model, is described by two variables: $x_{B j t}^{c}$ for charging and $x_{B j t}^{d}$ for discharging. Efficiencies are given by $0 \leq \eta_{B j}^{d} \leq 1$ and $0 \leq \eta_{B j}^{c} \leq 1$. We introduce variables $h_{B j t}$ to denote the amount of energy stored at node $j$ during time slot $t$. Storages have a total energy capacity related to its power output that again depends on $y_{B j}$. Storage balance must be fulfilled for every time step. Required constraints read as:

$$
\begin{array}{r}
0 \leq x_{B j t}^{d} \leq y_{B j}, \\
-y_{B j} \leq x_{B j t}^{c} \leq 0, \\
x_{B j t}=x_{B j t}^{d}+x_{B j t}^{c}, \\
h_{B j 0}=C A P_{B j 0} y_{B j}, \\
0 \leq h_{B j t} \leq w_{B j}^{\max }=C A P_{B j} y_{B j}, \\
h_{B j t}=h_{B j, t-1}-\frac{x_{B j t}^{d}}{\eta_{B j}^{d}}-x_{B j t}^{c} \eta_{B j}^{c} .
\end{array}
$$

Emissions from energy storages can be accounted for on a per-Megawatt basis or per-Energy-delivered basis. Depending on the modellers' target, either the use of resources or reduced life-time per cycle can be addressed through both parameters:

$$
e_{B j}=E_{B j}^{y} y_{B j}+\sum_{t \in T} E_{B j}^{x} x_{B j t}^{d} .
$$

\section{MODEL INPUT AND SCENARIO FRAMEWORK}

We choose a modified IEEE 118 bus network with corresponding input data and time series as a benchmark case that resembles conditions as can be found in northern Germany to determine energy system designs in a distant future of the year 2050 (Fig. 1] [20]. Investigating a greenfield approach to assess system designs of the future, the year has been chosen so that technical parameters could still be found in literature. The test case is modified to match the purpose of this study. In the original data set certain technologies are installed per node; fourfold the 'initial' capacity becomes the maximum expansion potential per technology. Coal and lignite options have been replaced by Gas power plants. Batteries can be expanded at any location with renewable technology potentials. Their maximal installed capacity is identical to the sum of renewable expansion potentials at the same node (Fig. 2). As a simplified approach to the (N-1) criterion, the line rating is capped at $70 \%$ of the actual thermal rating. Transmission expansion is restricted to the $380 \mathrm{kV}$ level and transformers; expansion candidates are twins of the original components that should be interpreted as thermal rating updates. More candidates as well as more sophisticated expansion options are possible, but beyond the intentions of this investigation.

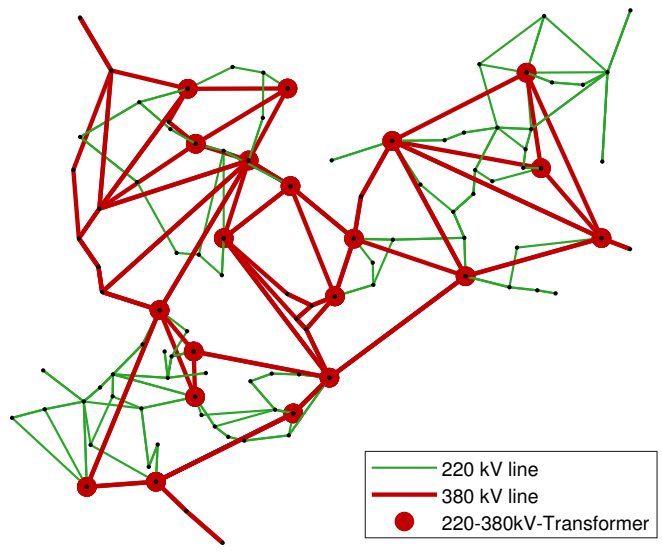

Fig. 1. Voltage levels on test grid

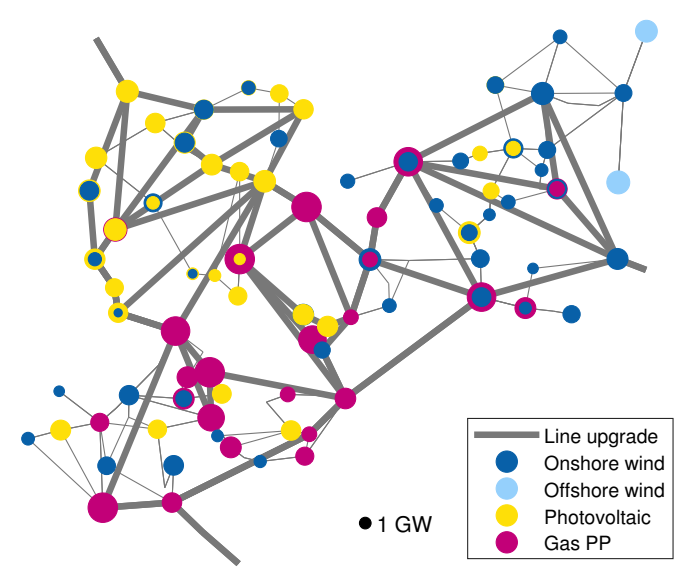

Fig. 2. Generation expansion potential (without batteries). Battery expansion potential is limited to the sum of renewable potential per node.

The assumed cost parameters in Table $\mathrm{V}$ have been adopted according to availability from the "Technology Data catalogue" published by the Danish Energy Agency [21], [22]. For studying the impact of life-cycle GHG parameters, four sensitivities are investigated. In the BASE sensitivity, emission parameters have been derived in accordance with the parameters outlined in Table []. Indirect emissions for Onshore and Offshore Wind relate to $4 \mathrm{~kg} / \mathrm{MWh}$, Photovoltaic is a bit higher with $6 \mathrm{~kg} / \mathrm{MWh}$. For the OMIT sensitivity indirect emissions are ignored to evaluate the impact of underestimating the full GWP of considered technologies. To underline the importance 
TABLE V

EXPANSION COST OF RENEWABLE TECHNOLOGIES AND THEIR EMISSION OUTPUTS IN FOUR SENSITIVITIES. CHANGES TO THE BASE SENSITIVITY ARE UNDERLINED.

\begin{tabular}{|c|c|c|c|c|c|c|c|c|c|c|c|}
\hline \multicolumn{4}{|c|}{ Expansion options } & \multicolumn{8}{|c|}{ Sensitivity: emissions in $\mathrm{CO}_{2}$-equivalents } \\
\hline Technology & Operation & Installation & Lifetime & BAs & & OMI & & BAT & & PHT & \\
\hline & [€/MWh] & {$[€ / \mathrm{kW}]$} & [a] & [kg/MWh] & [t/MW] & [kg/MWh] & [t/MW] & [kg/MWh] & [t/MW] & [kg/MWh] & [t/MW] \\
\hline Onshore Wind & 0.00 & 1439 & 30 & 0 & 300 & 0 & $\underline{0}$ & 0 & 300 & 0 & 300 \\
\hline Offshore Wind & 0.00 & 3106 & 30 & 0 & 500 & 0 & $\underline{0}$ & 0 & 500 & 0 & 500 \\
\hline Photovoltaic & 0.00 & 802 & 40 & 0 & 240 & 0 & $\underline{0}$ & 0 & 240 & 0 & $\underline{480}$ \\
\hline Gas power plant & 97.34 & 599 & 25 & 400 & 100 & 400 & $\underline{\overline{0}}$ & 400 & 100 & 400 & $\overline{100}$ \\
\hline Battery storage $^{a}$ & 1.60 & 510 & 30 & $10^{b}$ & 0 & $\underline{0}^{b}$ & $\overline{0}$ & $\underline{20}^{b}$ & 0 & $10^{b}$ & 0 \\
\hline Transformer & l & 34000 [€/MVA] & 50 & $100[\mathrm{t} / \mathrm{I}$ & [VA] & $\underline{0}[\overline{\mathrm{t} / \mathrm{M}}$ & $\mathrm{VA}]$ & $100 \overline{[\mathrm{t}} / \mathrm{N}$ & [VA] & $100[\mathrm{t} / \mathrm{I}$ & [VA] \\
\hline Line expansion & l & 1 Mio. $[€ / \mathrm{km}]$ & 50 & $100[\mathrm{t}$ & $\mathrm{km}]$ & $\underline{0}[\mathrm{t} / \mathrm{k}$ & & $100[\mathrm{t} /$ & $\mathrm{km}]$ & $100[\mathrm{t} /$ & $\mathrm{km}]$ \\
\hline
\end{tabular}

${ }^{a}$ Cycling efficiency: 0.92, energy-to-power-ratio: 2 [Wh/W]

${ }^{b}$ Per energy delivered

of low-emission storage, the GWP of batteries is doubled in the BATT sensitivity. Lastly, we double the indirect emissions of Photovoltaic in the PHTV sensitivity to investigate whether the emissions have a technology-dependent impact.

The sensitivities are run with 300 consecutive timesteps $\mathrm{s}^{2}$ To reflect the cost of investment, annuity cost - or rather the time-frame dependent shares of investment cost - are calculated with an interest rate of $0 \%$ and linear deprecation. Indirect emissions are taken into account in the same way. The emission cap is set to an annual target of $7 \mathrm{Mio}$. $\mathrm{tCO}_{2}$-eq or roughly $40 \mathrm{kgCO}_{2}$-eq/MWh, which corresponds to a emission reduction target of $95 \%$ in Germany in 2050.

\section{RESULTS AND DISCUSSION}

The resulting linear program consists of roundabout $5.2 \mathrm{E}+5$ variables and $7.8 \mathrm{E}+5$ constraints. It is solved by Gurobi's barrier method without crossover. The duality gap is below $0.3 \%$ in any case. As the problem is very decent still, it takes between 35 to 50 minutes to solve an instance on an Intel Core i7-7700HQ with four threads. Numerical results and locational decisions are summarised in Table VI.

In the BASE case (and any other sensitivity) photovoltaic is the dominant technology, being installed at its fully available capacity. Onshore wind and Battery storages are the second dominant technologies. Roughly 9.6 GW Gas power plants have been expanded with a capacity factor of $32 \%$. Grid expansion, for better comparison simply measured as total expansion costs, contributes $1.1 \%$ of the total system cost. It can be observed that Battery storage is expanded at any available location, correlated to the expansion of renewable technologies on site, providing additional flexibility and reducing grid congestion.

The OMIT sensitivity does not take indirect emissions into account. Although the emission cap has been fulfilled according to the sensitivity parameters, there are neglected indirect emissions that can be calculated in retrospect and compared to the BASE case. Thereby the emission cap has

\footnotetext{
${ }^{2}$ Hour 6301-6600 in scenario 2023B in the given dataset. The input time series have not been adjusted to expected load factors in 2050 and correspond to $12 \%, 25 \%$ and $43 \%$ for Photovoltaic, Onshore and Offshore wind in the given time frame. Wind options are therefore slightly underestimated; however, it does not change the message of the study.
}

been exceeded by more than $23 \%$, mostly due to the increased supply of Gas power plants, reducing the objective value by $9 \%$. At last, there is particularly less expansion of wind technologies in the north-east of the grid.

The BATT sensitivity increases indirect emissions of Battery cycling by $100 \%$. Counter-intuitively this leads to the actual expansion of Battery storage by $25 \%$. At the same time Gas power plant operation and installation decreases, resulting in an objective value 5\% higher compared to the BASE case. It is noticeable that also the grid expansion costs are rising, providing additional transmission capacity, which results in less curtailment of renewables.

Acknowledging the fact that photovoltaic is the dominant technology all of the former scenarios, its indirect emission parameter is doubled in the PHTV sensitivity. Apparently this has no effect on the expansion decisions for photovoltaic itself and instead becomes the more extreme version of the BATT sensitivity. Battery storage capacity increases by $38 \%$ compared to the BASE case (providing only $4 \%$ more discharge energy) and gas-fired plants appear even less ideal for the situation. Although the objective value reaches its maximum ( $+7 \%$ compare to BASE), grid expansion appears to be less valuable with even more Battery storage available.

Although it can hardly be generalized from this particular test case, the results indicate that the integration of lifecycle emissions into the equation of expansion models affects both, energy and technology mix. GHG emissions are significantly underestimated when omitting indirect emissions in the planning approach, which overestimates the role of conventional power plants and at the same time underestimates the necessity of renewable energy technologies. Increasing the GWP of certain technologies does not necessarily change the technology preference, but instead leads to a tighter emission budget and ultimately leads to different system designs.

To be able to make more generalized statements about how GWP changes G\&TEP modelling, further extensions are required. First of all, the test cases have only been calculated on 300 consecutive time steps. Solving the model on larger instances and for longer periods in reasonable time requires customized solving strategies and probably means of model reformulation and reduction. To answer questions about 
TABLE VI

EXPANSION RESULTS OF THE SCENARIOS AND COMPARED TO BASE CASE

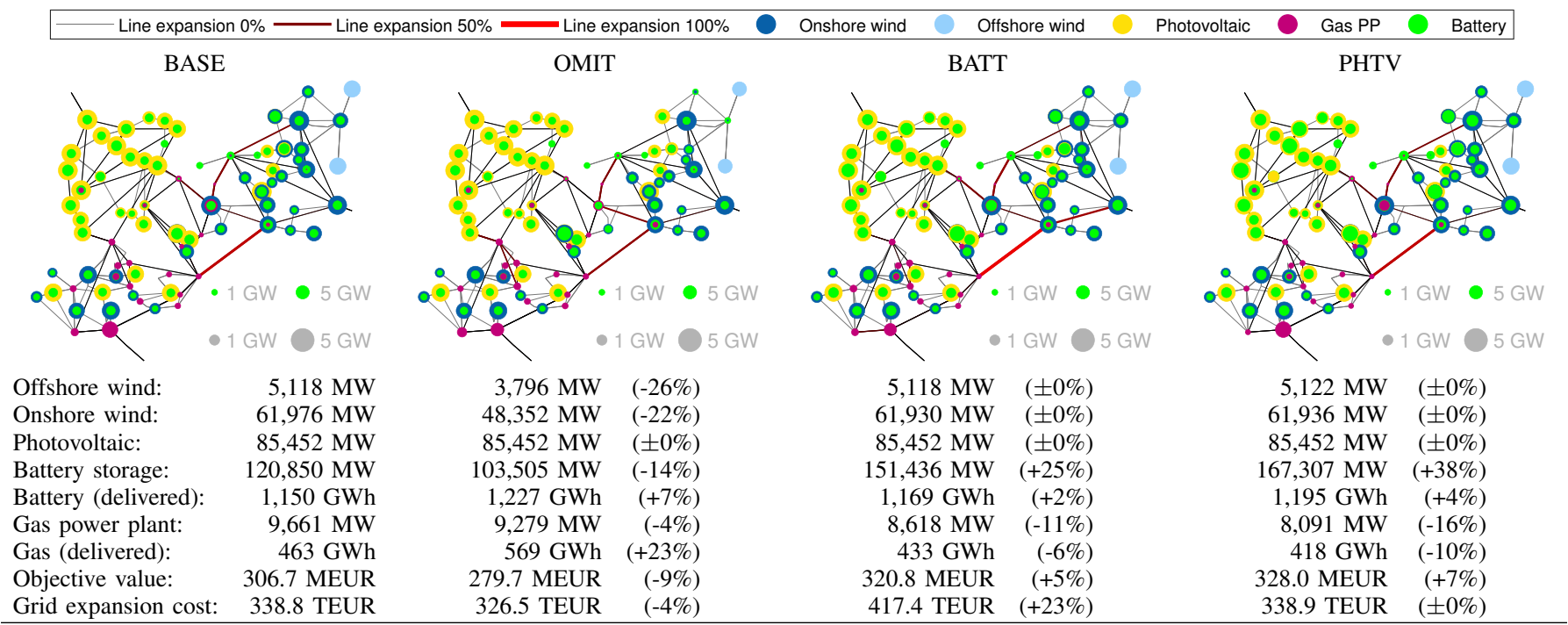

future energy systems, the planning approach cannot neglect the added flexibility and GHG reduction potentials of sector integration. Relevant technologies need to be integrated into the model to provide answers to more urgent questions. Lastly, additional research is required to better link the research in LCA and energy system modelling and to determine how the uncertainty of these parameters can be countered in future works.

\section{REFERENCES}

[1] H. Tekiner, D. W. Coit, and F. A. Felder, "Multi-period multi-objective electricity generation expansion planning problem with Monte-Carlo simulation," Electric Power Systems Research, vol. 80, no. 12, pp. 1394 $1405,2010$.

[2] H. Mavalizadeh and A. Ahmadi, "Hybrid expansion planning considering security and emission by augmented epsilon-constraint method," International Journal of Electrical Power \& Energy Systems, vol. 61, pp. $90-100,2014$.

[3] A. Rouhani, S. H. Hosseini, and M. Raoofat, "Composite generation and transmission expansion planning considering distributed generation," International Journal of Electrical Power \& Energy Systems, vol. 62, pp. 792-805, 2014.

[4] H. Sadeghi, M. Rashidinejad, and A. Abdollahi, "A comprehensive sequential review study through the generation expansion planning," Renewable and Sustainable Energy Reviews, vol. 67, pp. 1369-1394, 2017.

[5] F. Neumann and T. Brown, "The near-optimal feasible space of a renewable power system model," Electric Power Systems Research, vol. 190, no. 2, p. 106690, 2021.

[6] T. Junne, K.-K. Cao, K. K. Miskiw, H. Hottenroth, and T. Naegler, "Considering Life Cycle Greenhouse Gas Emissions in Power System Expansion Planning for Europe and North Africa Using Multi-Objective Optimization," Energies, vol. 14, no. 5, p. 1301, 2021.

[7] D. Weisser, "A guide to life-cycle greenhouse gas (GHG) emissions from electric supply technologies," Energy, vol. 32, no. 9, pp. 15431559, 2007.

[8] M. Pehl, A. Arvesen, F. Humpenöder, A. Popp, E. G. Hertwich, and G. Luderer, "Understanding future emissions from low-carbon power systems by integration of life-cycle assessment and integrated energy modelling," Nature Energy, vol. 2, no. 12, pp. 939-945, 2017.

[9] C. Unsihuay-Vila, J. W. Marangon-Lima, A. C. Zambroni de Souza, and I. J. Perez-Arriaga, "Multistage expansion planning of generation and interconnections with sustainable energy development criteria: A multiobjective model," International Journal of Electrical Power \& Energy Systems, vol. 33, no. 2, pp. 258-270, 2011.

[10] V. Kabakian, M. C. McManus, and H. Harajli, "Attributional life cycle assessment of mounted $1.8 \mathrm{kWp}$ monocrystalline photovoltaic system with batteries and comparison with fossil energy production system," Applied Energy, vol. 154, pp. 428-437, 2015.

[11] Á. Arcos-Vargas and L. Riviere, Grid Parity and Carbon Footprint. Cham: Springer International Publishing, 2019.

[12] J. Chipindula, V. Botlaguduru, H. Du, R. Kommalapati, and Z. Huque, "Life Cycle Environmental Impact of Onshore and Offshore Wind Farms in Texas," Sustainability, vol. 10, no. 6, p. 2022, 2018.

[13] J. K. Kaldellis and D. Apostolou, "Life cycle energy and carbon footprint of offshore wind energy. Comparison with onshore counterpart," Renewable Energy, vol. 108, pp. 72-84, 2017.

[14] M. Hiremath, K. Derendorf, and T. Vogt, "Comparative life cycle assessment of battery storage systems for stationary applications," Environmental science \& technology, vol. 49, no. 8, pp. 4825-4833, 2015.

[15] M. J. de Wild-Scholten, "Energy payback time and carbon footprint of commercial photovoltaic systems," Solar Energy Materials and Solar Cells, vol. 119, pp. 296-305, 2013.

[16] W. Wei, X. Wu, J. Li, X. Jiang, P. Zhang, S. Zhou, H. Zhu, H. Liu, H. Chen, J. Guo, and G. Chen, "Ultra-high voltage network induced energy cost and carbon emissions," Journal of Cleaner Production, vol. 178, pp. 276-292, 2018.

[17] A. Arvesen, R. N. Nes, D. Huertas-Hernando, and E. G. Hertwich, "Life cycle assessment of an offshore grid interconnecting wind farms and customers across the North Sea," The International Journal of Life Cycle Assessment, vol. 19, no. 4, pp. 826-837, 2014.

[18] G. P. Harrison, E. . J. Maclean, S. Karamanlis, and L. F. Ochoa, "Life cycle assessment of the transmission network in Great Britain," Energy Policy, vol. 38, no. 7, pp. 3622-3631, 2010.

[19] M. Rahmani, G. Hug, and A. Kargarian, "Comprehensive power transfer distribution factor model for large-scale transmission expansion planning," IET Generation, Transmission \& Distribution, vol. 10, no. 12, pp. 2981-2989, 2016.

[20] H. Barrios, A. Roehder, H. Natemeyer, and A. Schnettler, "A benchmark case for network expansion methods," in 2015 IEEE Eindhoven PowerTech, 2015, pp. 1-6.

[21] Danish Energy Agency and Energinet, "Technology Data: Generation of Electricity and District heating." [Online]. Available: https://ens.dk/sites/ens.dk/files/Statistik/technology_ data_catalogue_for_el_and_dh_-_0009.pdf

[22] —- "Technology Data: Energy storage." [Online]. Available: https://ens.dk/sites/ens.dk/files/Analyser/technology_data_ catalogue_for_energy_storage.pdf 